\title{
Substance Use by Adults with Medical Multimorbidity in the United States, 2015-2016
}

\author{
Benjamin H. Han, $M D^{1,2,3}$, Roxanne Ko, BA, BS ${ }^{4}$, and Joseph J. Palamar, $P h D^{2,3}$ \\ 'Department of Medicine, Division of Geriatric Medicine and Palliative Care, New York University School of Medicine, New York, NY, USA; \\ ${ }^{2}$ Department of Population Health, New York University Langone Medical Center, New York, NY, USA; ${ }^{3}$ Center for Drug Use and HIV/HCV Research, \\ NYU College of Global Public Health, New York, NY, USA; ${ }^{4}$ John A. Burns School of Medicine, University of Hawaii, Honolulu, HI, USA.
}

J Gen Intern Med 34(8):1394-6

DOI: $10.1007 / \mathrm{s} 11606-019-04980-1$

(c) Society of General Internal Medicine 2019

\section{INTRODUCTION}

Adults with medical multimorbidity, usually defined as $\geq 2$ concurrent chronic conditions, have high rates of healthcare utilization and often receive poorly coordinated care. ${ }^{1}$ Living with medical multimorbidity usually includes taking multiple medications and careful monitoring of individual diseases. The intersection of substance use and chronic disease is complex, ${ }^{2}$ as substance use can have negative effects on chronic diseases and its management.

However, few studies have focused on the intersection of chronic medical disease and substance use, especially among adults with multimorbidity. Therefore, the objective for this study was to use cross-sectional data from a nationally representative sample of adults in the United States (US) to estimate the prevalence of substance use among adults with chronic medical diseases, and to determine correlates of substance use among adults with medical multimorbidity.

\section{METHODS}

Data were utilized from the 2015-2016 National Survey on Drug Use and Health (NSDUH), an annual cross-sectional survey of non-institutionalized individuals in the US. Participants were assessed for past-year use of cannabis, cocaine, methamphetamine, heroin, inhalants, hallucinogens, and prescription drug misuse (opioids, tranquilizers, stimulants, and sedatives), and substance use disorder (SUD) related to use of these substances based on Diagnostic and Statistical Manual of Mental Disorders, Fourth Edition (DSM-IV) ${ }^{3}$ criteria. Ten self-reported chronic medical conditions (ever diagnosed by a doctor in one's lifetime) were examined (asthma, bronchitis/ COPD, cirrhosis, diabetes, heart conditions, hepatitis, high blood pressure, cancer, kidney disease, and HIV/AIDS). Prevalence of past-year substance use and SUD was estimated among adults ( $\geq 18$ years of age) with 0,1 , and $\geq 2$ chronic

This work has not been presented before.

Published online April 3, 2019 conditions. We utilized a Holm statistical correction ${ }^{4}$ for pastyear substance use to adjust for multiple comparisons. Bivariable and multivariable logistic regression models were used to examine correlates of past-year substance use among adults with $\geq 2$ chronic conditions including demographics, nicotine dependence, ${ }^{5}$ past-year all-cause emergency department use, and alcohol use disorder (AUD). ${ }^{3}$

\section{RESULTS}

The analytic sample included 85,701 participants. Table 1 presents past-year substance use and SUD comparing individuals with 0,1 , and $\geq 2$ chronic conditions. The most common substance used in the past year was cannabis, and cannabis use disorder was the most common SUD. Among adults with chronic conditions, past-year substance use was reported by $15.70 \%$ with 1 chronic condition and $12.80 \%$ with $\geq 2$ conditions, and substance use was higher among adults with no chronic conditions $(20.60 \%)$ ( $p<0.001$ for all comparisons). Criteria for any past-year SUD was met by $2.96 \%$ of adults with no chronic conditions, $2.61 \%$ with 1 chronic condition, and $2.29 \%$ with $\geq 2$ conditions $(p=0.01)$. Methamphetamine use disorder was significantly higher among those with $\geq 2$ chronic conditions.

Table 2 presents results from the logistic regression models for past-year drug use among adults with multimorbidity. Results from the adjusted model suggest that among adults with multimorbidity, odds of past-year drug use were lower among older participants (AOR 0.15 for adults $\geq 65$ [vs. those age 1834 ], $p<0.001$ ), females [vs. males] (AOR 0.78, $p=0.01$ ), and individuals with incomes $\geq \$ 75,000 /$ year [vs. $<\$ 20,000$ ] (AOR $0.75, p=0.04)$. Participants reporting nicotine dependence (AOR 2.51, $p<0.001$ ) or AUD (AOR 2.87, $p<0.001$ ) had higher odds of reporting past-year substance use.

\section{DISCUSSION}

While prevalence of past-year drug use was lower among adults with multimorbidity, nearly $13 \%$ of adults with multimorbidity reported past-year substance use. Given that substance use can negatively impact existing chronic medical disease and complicate its management, this has important 
Table 1 Past-Year Substance Use and Past-Year Substance Use Disorder by Number of Chronic Conditions, \% (n)—United States 2015-2016

\begin{tabular}{|c|c|c|c|c|c|c|c|c|c|}
\hline \multicolumn{10}{|c|}{ Number of chronic conditions* } \\
\hline \multirow{3}{*}{$\begin{array}{l}\text { Substance use } \\
\text { Any drug }\end{array}$} & \multicolumn{2}{|c|}{ Total $(n=85,701)$} & \multicolumn{2}{|c|}{$\begin{array}{l}\text { 0 chronic condition } \\
(n=58,299)\end{array}$} & \multicolumn{2}{|c|}{$\begin{array}{l}1 \text { chronic condition } \\
(n=19,487)\end{array}$} & \multicolumn{2}{|c|}{$\begin{array}{l}>2 \text { chronic conditions } \\
(n=7915)\end{array}$} & \multirow[t]{2}{*}{ value $^{*}$} \\
\hline & $n$ & $\%(95 \% \mathrm{CI})$ & $n$ & $\%(95 \% \mathrm{CI})$ & $n$ & $\%(95 \%$ CI $)$ & $n$ & $\%(95 \%$ CI $)$ & \\
\hline & 20,750 & $\begin{array}{l}18.10(17.70, \\
18.60)\end{array}$ & 15,010 & $\begin{array}{l}20.60(20.00 \\
21.10)\end{array}$ & 4366 & $\begin{array}{l}15.70(15.10 \\
16.40)\end{array}$ & 1374 & $\begin{array}{l}12.80(11.70 \\
14.00)\end{array}$ & $<0.001$ \\
\hline $\begin{array}{l}\text { Any drug, excluding } \\
\text { cannabis }\end{array}$ & 10,447 & $\begin{array}{l}9.13(8.84, \\
9.42)\end{array}$ & 7449 & $\begin{array}{l}10.20(9.84 \\
10.60)\end{array}$ & 2256 & $\begin{array}{l}8.02(7.57 \\
8.49)\end{array}$ & 742 & $\begin{array}{l}6.95(6.30 \\
7.65)\end{array}$ & $<0.001$ \\
\hline Cannabis & 16,764 & $\begin{array}{l}13.90(13.50, \\
14.30)\end{array}$ & 12,389 & $\begin{array}{l}16.40(15.90, \\
16.90)\end{array}$ & 3382 & $\begin{array}{l}11.50(10.90, \\
12.10)\end{array}$ & 993 & $\begin{array}{l}8.47(7.58 \\
9.45)\end{array}$ & $<0.001$ \\
\hline Cocaine & 2386 & $\begin{array}{l}1.97(1.84, \\
2.12)\end{array}$ & 1790 & $\begin{array}{l}2.42(2.27 \\
2.58)\end{array}$ & 494 & $\begin{array}{l}1.67(1.43, \\
1.96)\end{array}$ & 102 & $\begin{array}{l}0.75(0.58 \\
0.97)\end{array}$ & $<0.001$ \\
\hline Methamphetamine & 686 & $\begin{array}{l}0.61(0.55 \\
0.67)\end{array}$ & 406 & $\begin{array}{l}0.54(0.47 \\
0.61)\end{array}$ & 180 & $\begin{array}{l}0.63(0.50 \\
0.80)\end{array}$ & 100 & $\begin{array}{l}0.84(0.65 \\
1.10)\end{array}$ & 0.02 \\
\hline Inhalants & 595 & $\begin{array}{l}0.45(0.40 \\
0.52)\end{array}$ & 423 & $\begin{array}{l}0.51(0.44 \\
0.58)\end{array}$ & 137 & $\begin{array}{l}0.42(0.33 \\
0.55)\end{array}$ & 35 & $\begin{array}{l}0.30(0.17 \\
0.51\end{array}$ & 0.09 \\
\hline Heroin & 446 & $\begin{array}{l}0.36(0.31 \\
0.40)\end{array}$ & 309 & $\begin{array}{l}0.39(0.35 \\
0.44)\end{array}$ & 91 & $\begin{array}{l}0.28(0.20 \\
0.40)\end{array}$ & 46 & $\begin{array}{l}0.35(0.22 \\
0.54)\end{array}$ & 0.22 \\
\hline $\begin{array}{l}\text { Prescription opioid } \\
\text { misuse }\end{array}$ & 4945 & $\begin{array}{l}4.55(4.36 \\
4.74)\end{array}$ & 3392 & $\begin{array}{l}4.83(4.59, \\
5.09)\end{array}$ & 1119 & $\begin{array}{l}4.23(3.90 \\
4.60)\end{array}$ & 434 & $\begin{array}{l}4.00(3.53 \\
4.54)\end{array}$ & 0.004 \\
\hline Hallucinogens & 2598 & $\begin{array}{l}1.79(1.66 \\
1.93)\end{array}$ & 2019 & $\begin{array}{l}2.28(2.13 \\
2.44)\end{array}$ & 504 & $\begin{array}{l}1.42(1.21 \\
1.65)\end{array}$ & 75 & $\begin{array}{l}0.53(0.35 \\
0.82)\end{array}$ & $<0.001$ \\
\hline $\begin{array}{l}\text { Prescription tranquilizer } \\
\text { misuse }\end{array}$ & 2696 & $\begin{array}{l}2.34(2.21 \\
2.48)\end{array}$ & 1831 & $\begin{array}{l}2.49(2.33 \\
2.65)\end{array}$ & 632 & $\begin{array}{l}2.11(1.88 \\
2.36)\end{array}$ & 233 & $\begin{array}{l}2.18(1.81 \\
2.63)\end{array}$ & 0.05 \\
\hline $\begin{array}{l}\text { Prescription sedative } \\
\text { misuse }\end{array}$ & 559 & $\begin{array}{l}0.60(0.54 \\
0.67)\end{array}$ & 338 & $\begin{array}{l}0.54(0.48 \\
0.62)\end{array}$ & 145 & $\begin{array}{l}0.58(0.46 \\
0.73)\end{array}$ & 76 & $\begin{array}{l}0.88(0.66, \\
1.16)\end{array}$ & 0.01 \\
\hline $\begin{array}{l}\text { Prescription stimulant } \\
\text { misuse }\end{array}$ & 7489 & $\begin{array}{l}6.70(6.45 \\
6.96)\end{array}$ & 5213 & $\begin{array}{l}7.15(6.84 \\
7.48)\end{array}$ & 1663 & $\begin{array}{l}6.09(5.69 \\
6.51)\end{array}$ & 613 & $\begin{array}{l}6.04(5.45 \\
6.69)\end{array}$ & $<0.001$ \\
\hline$\geq 2$ drug use & 8320 & $\begin{array}{l}6.67(6.47, \\
6.87)\end{array}$ & 6039 & $\begin{array}{l}7.75(7.49 \\
8.02)\end{array}$ & 1743 & $\begin{array}{l}5.56(5.18 \\
5.97)\end{array}$ & 538 & $\begin{array}{l}4.37(3.92 . \\
4.87)\end{array}$ & $<0.001$ \\
\hline Substance use disorder ${ }^{\dagger}$ & $n$ & $\%(95 \% \mathrm{CI})$ & $n$ & $\%(95 \% \mathrm{CI})$ & $n$ & $\%(95 \% \mathrm{CI})$ & $n$ & $\%(95 \% \mathrm{CI})$ & \\
\hline Any drug & 3459 & $\begin{array}{l}2.77(2.63 \\
2.91)\end{array}$ & 2370 & $\begin{array}{l}2.96(2.81 \\
3.12)\end{array}$ & 803 & $\begin{array}{l}2.61(2.33 \\
2.92)\end{array}$ & 286 & $\begin{array}{l}2.29(1.94 \\
2.71)\end{array}$ & 0.01 \\
\hline $\begin{array}{l}\text { Any drug, excluding } \\
\text { cannabis }\end{array}$ & 1851 & $\begin{array}{l}1.64(1.53, \\
1.75)\end{array}$ & 1146 & $\begin{array}{l}1.54(1.42, \\
1.67)\end{array}$ & 489 & $\begin{array}{l}1.74(1.54, \\
1.96)\end{array}$ & 216 & $\begin{array}{l}1.85(1.55, \\
2.20)\end{array}$ & 0.07 \\
\hline Cannabis & 1960 & $\begin{array}{l}1.39(1.30 \\
1.49)\end{array}$ & 1454 & $\begin{array}{l}1.69(1.57, \\
1.81)\end{array}$ & 412 & $\begin{array}{l}1.16(0.99, \\
1.36)\end{array}$ & 94 & $\begin{array}{l}0.65(0.48 \\
0.87)\end{array}$ & $<0.001$ \\
\hline Cocaine & 364 & $\begin{array}{l}0.35(0.30 \\
0.42)\end{array}$ & 244 & $\begin{array}{l}0.36(0.30 \\
0.43)\end{array}$ & 89 & $\begin{array}{l}0.39(0.29 \\
0.53)\end{array}$ & 31 & $\begin{array}{l}0.26(0.15 \\
0.50)\end{array}$ & 0.31 \\
\hline Inhalants & 30 & $\begin{array}{l}0.02(0.01 \\
0.04)\end{array}$ & 19 & $\begin{array}{l}0.03(0.01 \\
0.05)\end{array}$ & 6 & $\begin{array}{l}0.01(0.01 \\
0.04)\end{array}$ & 5 & $\begin{array}{l}0.04(0.01 \\
0.16)\end{array}$ & 0.47 \\
\hline Methamphetamine & 350 & $\begin{array}{l}0.30(0.25 \\
0.35)\end{array}$ & 193 & $\begin{array}{l}0.23(0.19 \\
0.28)\end{array}$ & 100 & $\begin{array}{l}0.35 \\
0.48)\end{array}$ & 57 & $\begin{array}{l}0.46(0.33 \\
0.65)\end{array}$ & 0.004 \\
\hline Heroin & 314 & $\begin{array}{l}0.24(0.21 \\
0.28)\end{array}$ & 218 & $\begin{array}{l}0.27(0.24 \\
0.32)\end{array}$ & 66 & $\begin{array}{l}0.20(0.14 \\
0.29)\end{array}$ & 30 & $\begin{array}{l}0.21(0.13 \\
0.32)\end{array}$ & 0.19 \\
\hline Prescription opioid & 805 & $\begin{array}{l}0.75(0.68 \\
0.83)\end{array}$ & 477 & $\begin{array}{l}0.67(0.60 \\
0.76)\end{array}$ & 224 & $\begin{array}{l}0.81(0.69 \\
0.96)\end{array}$ & 104 & $\begin{array}{l}0.95(0.75 \\
1.22)\end{array}$ & 0.02 \\
\hline Hallucinogens & 159 & $\begin{array}{l}0.10(0.08 \\
0.13)\end{array}$ & 98 & $\begin{array}{l}0.10(0.08, \\
0.14)\end{array}$ & 52 & $\begin{array}{l}0.12(0.09, \\
0.17)\end{array}$ & 9 & $\begin{array}{l}0.05(0.02, \\
0.20)\end{array}$ & 0.33 \\
\hline Prescription tranquilizer & 275 & $\begin{array}{l}0.24(0.20 \\
0.28)\end{array}$ & 163 & $\begin{array}{l}0.21(0.17 \\
0.26)\end{array}$ & 76 & $\begin{array}{l}0.25(0.19 \\
0.33)\end{array}$ & 36 & $\begin{array}{l}0.31(0.20 \\
0.47)\end{array}$ & 0.18 \\
\hline Prescription sedative & 64 & $\begin{array}{l}0.05(0.04 \\
0.07)\end{array}$ & 29 & $\begin{array}{l}0.04(0.02 \\
0.06)\end{array}$ & 23 & $\begin{array}{l}0.07(0.04 \\
0.11)\end{array}$ & 12 & $\begin{array}{l}0.10(0.04, \\
0.23)\end{array}$ & 0.12 \\
\hline Prescription stimulant & 245 & $\begin{array}{l}0.19(0.16 \\
0.23)\end{array}$ & 143 & $\begin{array}{l}0.17(0.14, \\
0.21)\end{array}$ & 74 & $\begin{array}{l}0.22 \\
0.30)\end{array}$ & 28 & $\begin{array}{l}0.22(0.13, \\
0.40)\end{array}$ & 0.34 \\
\hline
\end{tabular}

All percentages are weighted

*The National Survey on Drug Use and Health queries 10 major chronic conditions that include heart conditions, diabetes, chronic bronchitis/chronic obstructive pulmonary disease, cirrhosis, hepatitis B or C, kidney

disease, asthma, HIV/AIDS, cancer, and high blood pressure (hypertension)
$\forall$ Based on the Diagnostic and Statistical Manual of Mental Disorders, Fourth Edition 3

$\not$ We utilized a Holm statistical correction 4 for past-year substance use and substance use disorder variables. Although we present unadjusted p values, methamphetamine use and sedative misuse only approached significance given the correction so we do not interpret these two tests as being significant. For substance use disorder, only cannabis and methamphetamine use disorders remained significant with $p<0.05$ after correction

public health implications. The lower prevalence of substance use among adults with multimorbidity may be because younger adults are less likely to have chronic conditions, and some of those with multimorbidity may have stopped using substance due to illness. ${ }^{6}$

These results identify a potentially high-risk population with medical multimorbidity and polysubstance use (substance, AUD, and/or tobacco dependence), which has important health implications. The finding of higher prevalence of methamphetamine use disorder among adults with multimorbidity requires further study and is not described in the literature. Substance use and substance use disorder among adults with multimorbidity also needs to be better explored by disease type. 
Table 2 Correlates of Past-Year Substance Use Among Adults with Multimorbidity-United States 2015-2016

\begin{tabular}{|c|c|c|c|c|c|c|}
\hline Characteristic & Odds ratio & $(95 \% \mathrm{CI})$ & $p$ value & Adjusted odds ratio & $(95 \% \mathrm{CI})$ & $p$ value \\
\hline \multicolumn{7}{|l|}{ Age group } \\
\hline $18-34$ & 1.00 & & & 1.00 & & \\
\hline $35-49$ & 0.44 & $(0.37,0.52)$ & $<0.001$ & 0.51 & $(0.41,0.61)$ & $<0.001$ \\
\hline $50-64$ & 0.29 & $(0.24,0.35)$ & $<0.001$ & 0.37 & $(0.29,0.49)$ & $<0.001$ \\
\hline$\geq 65$ & 0.09 & $(0.07,0.13)$ & $<0.001$ & 0.15 & $(0.11,0.22)$ & $<0.001$ \\
\hline \multicolumn{7}{|l|}{ Sex } \\
\hline Male & 1.00 & & & 1.00 & & \\
\hline Female & 0.86 & $(0.74,1.02)$ & 0.08 & 0.78 & $(0.64,0.95)$ & 0.01 \\
\hline \multicolumn{7}{|l|}{ Race/ethnicity } \\
\hline Non-Hispanic White & 1.00 & & & 1.00 & & \\
\hline Non-Hispanic Black/African American & 1.27 & $(0.97,1.66)$ & 0.09 & 0.99 & $(0.75,1.30)$ & 0.94 \\
\hline Hispanic & 1.15 & $(0.88,1.52)$ & 0.30 & 0.84 & $(0.63,1.13)$ & 0.25 \\
\hline Non-Hispanic Asian & 0.86 & $(0.36,2.01)$ & 0.72 & 0.78 & $(0.33,1.87)$ & 0.58 \\
\hline Other & 1.31 & $(0.94,1.82)$ & 0.11 & 1.02 & $(0.74,1.42)$ & 0.89 \\
\hline \multicolumn{7}{|l|}{ Total family income } \\
\hline$<\$ 20,000$ & 1.00 & & & 1.00 & & \\
\hline$\$ 20,000-\$ 49,999$ & 0.59 & $(0.47,0.73)$ & $<0.001$ & 0.81 & $(0.62,1.05)$ & 0.11 \\
\hline$\$ 50,000-\$ 74,999$ & 0.49 & $(0.35,0.69)$ & $<0.001$ & 0.73 & $(0.51,1.04)$ & 0.08 \\
\hline$\geq \$ 75,000$ & 0.51 & $(0.41,0.64)$ & $<0.001$ & 0.75 & $(0.57,0.98)$ & 0.04 \\
\hline \multicolumn{7}{|l|}{ Marital status } \\
\hline Married & 1.00 & & & 1.00 & & \\
\hline Widowed & 0.61 & $(0.41,0.89)$ & 0.01 & 0.89 & $(0.59,1.34)$ & 0.56 \\
\hline Divorced or separated & 2.23 & $(1.73,2.87)$ & $<0.001$ & 1.70 & $(1.27,2.28)$ & 0.001 \\
\hline Never married & 4.05 & $(3.18,5.15)$ & $<0.001$ & 1.98 & $(1.45,2.68)$ & $<0.001$ \\
\hline \multicolumn{7}{|l|}{ Tobacco use } \\
\hline Nicotine dependence* & 3.82 & $(3.22,4.54)$ & $<0.001$ & 2.51 & $(2.04,3.09)$ & $<0.001$ \\
\hline \multicolumn{7}{|l|}{ Alcohol use } \\
\hline Alcohol use disorder ${ }^{\dagger}$ & 4.49 & $(3.48,5.80)$ & $<0.001$ & 2.87 & $(2.22,3.72)$ & $<0.001$ \\
\hline \multicolumn{7}{|l|}{ Healthcare utilization } \\
\hline All-cause ER use past year & 1.34 & $(1.16,1.55)$ & $<0.001$ & 1.06 & $(0.90,1.26)$ & 0.49 \\
\hline \multicolumn{7}{|l|}{ Number of chronic conditions } \\
\hline 2 conditions & 1.00 & & & & & \\
\hline 3-4 conditions & 0.97 & $(0.82,1.15)$ & 0.70 & 1.16 & $(0.97,1.39)$ & 0.11 \\
\hline$\geq 5$ conditions & 0.57 & $(0.36,0.91)$ & 0.02 & 0.67 & $(0.39,1.15)$ & 0.15 \\
\hline
\end{tabular}

Multimorbidity defined as 2 or more of the following heart conditions, diabetes, chronic bronchitis/chronic obstructive pulmonary disease, cirrhosis, hepatitis B or C, kidney disease, asthma, HIV/AIDS, cancer, and high blood pressure (hypertension)

$*$ Based on the Nicotine Dependence Syndrome Scale $(N D S S)^{5}$
$\forall$ Based on the Diagnostic and Statistical Manual of Mental Disorders, Fourth Edition 3

Substance use adds complexity to the already-challenging care of adults with multimorbidity. Adults with multimorbidity should be screened for substance use, and those with the compound comorbidity of SUD and medical disease require integrated, patient-centered approaches to care. ${ }^{2}$

Acknowledgements: The authors would like to thank Charles $M$. Cleland, $\mathrm{PhD}$, for his statistical advice and mentorship on the revision for this paper.

Corresponding Author: Benjamin H. Han, MD; Department of Medicine, Division of Geriatric Medicine and Palliative Care New York University School of Medicine, New York, NY, USA (e-mail: Benjamin. Han@nyumc.org).

Funding Information This research was funded by the following grants through the National Institutes of Health: K23DA043651 (Han) and K01DA038800 from the National Institute on Drug Abuse (Palamar) and T35AG050998-01 from the National Institute on Aging (Ko). The National Institutes of Health provided financial support for the project and the preparation of the manuscript but did not have a role in the design of the study, the analysis of the data, the writing of the manuscript, nor the decision to submit the present research.

\section{Compliance with Ethical Standards:}

Conflict of Interest: The authors declare that they do not have a conflict of interest.
Disclaimer: The content is solely the responsibility of the authors and does not necessarily represent the official views of the National Institutes of Health.

\section{REFERENCES}

1. Boyd CM, Fortin M: Future of multimorbidity research: how should understanding of multimorbidity inform health system design?, Public Health Rev. 2010;32:451-474.

2. Han, BH. Aging, multimorbidity, and substance use disorders: The growing case for integrating the principles of geriatric care and harm reduction. Int J Drug Policy. 2018;58:135-136.

3. American Psychiatric Association. Diagnostic and Statistical Manual of Mental Disorders. 4th ed. Washington, DC: American Psychiatric Association; 1994.

4. Holm S. A simple sequentially rejective multiple test procedure. Scand J Stat 1979;6:65-70.

5. Shiffman S, Waters A, Hickcox M. The nicotine dependence syndrome scale: a multidimensional measure of nicotine dependence. Nicotine Tob Res 2004; 6:327-348.

6. Naimi TS, Stockwell T, Zhao J, et al. Selection biases in observationa studies affect associations between 'moderate' alcohol consumption and mortality. Addiction 2017;112:207-214.

Publisher's Note: Springer Nature remains neutral with regard to jurisdictional claims in published maps and institutional affiliations. 